\title{
Is there an indication left for gastric band? A single center experience on 178 patients with a follow-up of 10 years
}

\author{
Antonio Vitiello ${ }^{1}$ (D) $\cdot$ Giovanna Berardi $^{1} \cdot$ Nunzio Velotti $^{1} \cdot$ Giovanni Domenico De Palma $^{2} \cdot$ Mario Musella $^{1}$
}

Received: 15 June 2020 / Accepted: 23 July 2020 / Published online: 31 July 2020

(c) The Author(s) 2020

\begin{abstract}
Background Laparoscopic Adjustable Gastric Banding (LAGB) has been widely performed in the past at our university bariatric center. Aim of this study was to retrospectively assess long term outcomes of LAGB at our university hospital, with special regard to non-response $(\mathrm{EWL}<25 \%)$ and rate of band removal.

Methods Retrospective search of prospectively maintained database of our university bariatric center was carried out to find all consecutive patients that had undergone LAGB at our department with a minimum follow-up of 10 years. Collected data were sex, age, body mass index (BMI), obesity related diseases remission, complications and weight loss.

Results After 10 years, patients with the band $(n=144)$ in place had a BMI of $35.2 \pm 7.5 \mathrm{~kg} / \mathrm{m} 2$, while $\% \mathrm{EWL}$ and $\%$ TWL were $40.8 \pm 52.4$ and $18.9 \pm 20.7$. Seventy-four $(41.6 \%)$ achieved a success $(\%$ EWL $>50)$, while $38(21.3 \%)$ were non-responders (\%EWL <25), $32(18 \%)$ had an insufficient weight loss $(25<\%$ EWL < 50) and $34(19.1 \%)$ underwent band removal. Among these, $6(3.4 \%)$ were removed for complications and 28 (15.7\%) for insufficient weight loss. Weight regain occurred in 38 out of 144 (26.4\%) subjects with the band in place at 10 years. Only one case of early vomiting with readmission for medical treatment was recorded. Slippage, erosion/migration and port/tube complications occurred in 4 (2.2\%), $2(1.1 \%)$ and $9(5 \%)$ cases respectively.

Conclusion LAGB is a safe and moderately effective bariatric procedure but it showed disappointing rates of removal, non-response and remission from comorbidities. However, LAGB could still be proposed for selected/motivated patients.
\end{abstract}

Keywords LAGB $\cdot$ Gastric band $\cdot$ Band removal $\cdot$ Long term results

Antonio Vitiello

antoniovitiello_@hotmail.it

Giovanna Berardi

giovannaberardi88@gmail.com

Nunzio Velotti

nunzio.velotti@gmail.com

Giovanni Domenico De Palma

giovannidomenico.depalma@unina.it

Mario Musella

mario.musella@unina.it

1 Advanced Biomedical Sciences Department, Naples

"Federico II" University, AOU "Federico II", Via S. Pansini

5, 80131 Naples, Italy

2 Department of Clinical Medicine and Surgery, University of Naples Federico II, Naples, Italy

\section{Introduction}

Laparoscopic approach for gastric banding not only represented a milestone for the history of this procedure but also promoted the endorsement and diffusion of bariatric surgery itself. Laparoscopic adjustable gastric banding (LAGB) became one of the most performed bariatric procedures accounting for $24.4 \%$ of all bariatric procedures worldwide in 2003 and $42.3 \%$ in $2008[1,2]$.

However, several studies published in the subsequent years demonstrated a long-term failure rate ranging from 40 to $70 \%$ [3-6]. Indeed, in 2011 percentage of LAGBs performed worldwide decreased to $17.8 \%$, mainly because of the success of the laparoscopic sleeve gastrectomy (LSG) [7]. In the last years, the decline of LAGB has continued and, according to last IFSO reports (International Federation for Surgery for Obesity) [8, 9], it has been almost abandoned. 
More than 10 years ago, LAGB was the most performed procedure at our Center for the Interdisciplinary Treatment of Obesity, accounting for $50 \%$ of all bariatric surgeries, but currently LSG and OAGB/MGB are the preferred surgical choices. Currently, about two hundred interventions are performed each year in our Institution and LAGB represents only $5-10 \%$.

Aim of this study was to retrospectively assess long term outcomes of LAGB at our university hospital, with special regard to non-response $(\mathrm{EWL}<25 \%)$ and rate of band removal.

\section{Materials and methods}

Retrospective search of prospectively maintained database of our university bariatric center was carried out to find all consecutive patients that had undergone LAGB at our department with a minimum follow-up of 10 years. Inclusion criteria were age between 18 and 60 years, BMI $>40 \mathrm{~kg} / \mathrm{m}^{2}$ or $>35$ with a related disease. Subjects with previous history of bariatric or abdominal surgery were excluded.

Collected data were sex, age, body mass index (BMI), obesity related diseases remission, complications and weight loss.

\section{Surgical technique}

All patients were positioned on the operating table in a 15-30 degrees reverse Trendelenburg position; the surgeon stood between the patient legs; the cameraman was on the left. Closed pneumoperitoneum of 12-14 mm Hg was achieved using a Veress needle. A total number of 4 trocars were placed as follows: one $10 \mathrm{~mm}$ trocar above the umbilicus for the 30 degrees laparoscope; another $10 \mathrm{~mm}$ trocar on the midclavicular line 5-6 cm below the costal margin; one $5 \mathrm{~mm}$ trocar was placed below the xiphoid appendices for liver retraction and a $5 \mathrm{~mm}$ trocar was placed between the right anterior axillary and the midclavicular line, $4-5 \mathrm{~cm}$ subcostally. The operation then started with the dissection of the gastrophrenic ligament and with the opening of the pars flaccida of the small omentum. A grasper was moved along the right crus to create a retrogastric tunnel; a bandplacer was then inserted in this path to appear on the greater curvature of the stomach at the site of the prior dissection of His angle. The band was drawn along this tunnel and then closed. The procedure ended with 2 gastro-gastric sero-serous nonabsorbable sutures passed between the gastric fundus and the gastric pouch above the band. Leak test was not performed routinely, and nasogastric tube was not placed [10].

\section{Preoperative evaluation and follow-up}

All patients were preoperatively evaluated by an interdisciplinary team consisting of endocrinologists, psychiatrists, dieticians and surgeons. Liquid diet was started on postoperative day 1 and discharge was planned the day after for uneventful procedures. Pureed foods were allowed after postoperative day 15 and normal diet after 30 days. Follow-up appointments were routinely planned at 1, 3, 6 and 12 months. After the first year, visits were planned every 6 months. Band regulations were decided on the base of patient's symptoms and weight. Unplanned appointments and regulations were scheduled in case of acute vomiting or dysphagia and a barium swallow was required.

\section{Weight loss}

Weight loss was calculated as percentage of excess weight loss (\%EWL) at 1, 5 and 10 years.

Success at 10 years at was defined as $\% E W L \geq 50$, nonresponse was set as \%EWL $<25$ [11], while $25<\% \mathrm{EWL}<50$ was set as insufficient weight loss (IWL). Weight regain at 10 years was defined as $\% \mathrm{EWL}<50$ for a patient who had previously achieved $\%$ EWL $>50$.

In addition, total weight loss percent (\%TWL) was calculated. Multiple linear regression using preoperative characteristics (sex, age, BMI and comorbidities) as independent variables and \% TWL at 10 years as dependent variable was performed.

\section{Remission from obesity related disease}

Remission of type 2 diabetes (T2DM) was considered as a value of glycated haemoglobin A1c (HbA1C) $<6.5 \%$ off antidiabetic medications [12]. Hypertension (HTN) remission was defined as blood pressure $<140 / 90$ with off antihypertensive medication [13]. Dyslipidaemia cut-offs points were chosen according to the American Heart Association criteria to identify metabolic syndrome [14].

\section{Complications}

Early postoperative ( $<30$ days) complications (bleeding, perforation, untreatable vomiting) and late complications (slippage, erosion/migration and port/tube infection) were recorded.

\section{Statistical analysis}

Statistical analysis was performed using Statistical Package for Social Sciences (SPSS) software for windows. 
Continuous data are expressed as means \pm standard deviation. Significance was set at a $p$ value of $<0.05$.

\section{Results}

A total number of 225 patients were eligible for this study, but $47(21 \%)$ subjects were loss during follow up; therefore 178 (79\%; 52 males/126 females) patients were included. Initial age and BMI were $38 \pm 11.5$ years and $44.4 \pm 6.5 \mathrm{~kg} /$ $\mathrm{m}^{2}$ respectively.

\section{Weight loss}

After 10 years, patients with the band in place $(n=144)$ had a mean BMI of $35.2 \pm 7.5 \mathrm{~kg} / \mathrm{m}^{2}$, while $\%$ EWL and \% TWL were $40.8 \pm 52.4$ and $18.9 \pm 20.7$. Seventy-four $(41.6 \%)$ achieved a success $(\% \mathrm{EWL}>50)$, while 38 (21.3\%) were non-responders $(\% \mathrm{EWL}<25), 32(18 \%)$ had an insufficient weight loss $(25<\% \mathrm{EWL}<50)$ and $34(19.1 \%)$ underwent band removal.

Weight regain occurred in 38 out of 144 (26.4\%) subjects with the band in place at 10 years. Multiple regression analysis demonstrated a significant relationship in favor of male gender $(p=0.01)$ and BMI $(p<0.01)$ with \%TWL at 10 years. \%EWL, \% TWL and number of band removals during follow-up are pictured in Fig. 1.

\section{Remission from obesity related disease}

At baseline 35 (19.7\%) patients were diagnosed with Hypertension, 20 (11.2\%) were diabetic and 34 (19.1\%) had dyslipidaemia. After 10 years, remission rate from HTN, T2DM and Dyslipidaemia were $20 \%(n=7), 0 \%(n=0)$ and $23.5 \%$ $(n=8)$ respectively.

\section{Complications}

One patient was readmitted on postoperative day 4 for severe vomiting and discharged after medical treatment; no other early complication was found in our cohort. Slippage, erosion/migration and port/tube complications occurred in 4 $(2.2 \%), 2(1.1 \%)$ and $9(5 \%)$ cases respectively. Port/tube problems were resolved with minor interventions in local anaesthesia.

Band removal rate at 10 years was $19.1 \%(n=34), 6$ (3.4\%) were removed for complications and 28 (15.7\%) for insufficient weight loss. Among these, 24 subjects refused further treatment while 10 were converted to other procedures. Six patients were converted to LSG, 2 to Roux-en-Y gastric bypass (RYGB) and 2 to Mini-bypass/One anastomosis gastric bypass (MGB/OAGB). All conversions were performed in one step.

\section{Discussion}

LAGB has always been considered as a definitive procedure due to its adjustable nature and high-tolerated material. Silicone is, indeed, the best-tolerated material, and recent bands are also designed to create few gastric adhesions [15]. However, the gastric band remains a foreign body and with time it may migrate or slip. Also, the port-tube system, which is placed in the subcutaneous tissue, could get infected and migrate due to multiple traumas of regulations. However, infection, migration and slippage in large series are reported in less than $5 \%$ of cases [16-18], being unsatisfactory weight loss the major reason for removal or conversion to another procedure [18, 19]. Interestingly, several studies have demonstrated effectiveness and safety of conversion of LAGB to other bariatric procedures in one- or two-steps [20-22].

Main reason of the decline of LAGB is the unsatisfactory weight loss due to non-response or weight regain, as
Fig. 1 Trend of percentage of excess weight loss (\%EWL) and Total Weight Loss percent (\%TWL) during follow-up. $n=$ number of removals

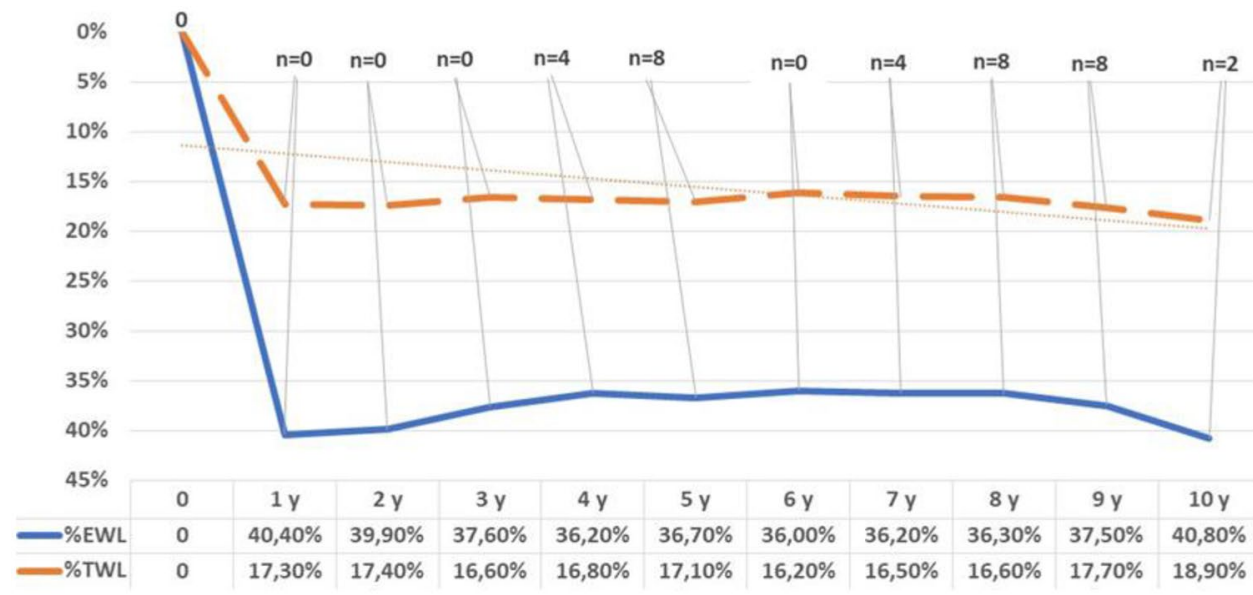


reported in series with medium [23] or long term [24-26] follow-up. Nevertheless, a study with 18 years of follow-up reported that despite band removal may occur in half of the patients over time, considering its reversibility and safety, LAGB still has a place in the treatment of morbid obesity [27].

A recent systematic review for studies with 10-year follow-up data, including a total of 9706 patients [28] showed a mean of $49 \%$ EWL at 10 years with a $30 \%$ band removal rate. However, reported failure and removal rates for LAGB reached $70 \%$ in studies with a follow-up longer than 20 years [29-32].

These data are consistent with our findings; $60 \%$ of our patients underwent band removal or did not achieve $\%$ EWL $>50$. However, $40 \%$ of subjects with the band in place still had a success after 10 years and this outcome could demonstrate that LAGB may be effective for selected/ motivated patients in medium and in long term. Conversely to previous evidences [33-35], LAGB proved to be more effective in male patients and in those with higher BMI. Moreover, weight regain (WR) occurred in $26 \%$ of cases, which is comparable to WR after other bariatric interventions such as LSG [36] or RYGB [37, 38]. Removal rate was slightly less than $20 \%$ in our cohort and a systematic review [39] has shown that all procedures have a substantial need for re-operative surgery and the levels of reoperation for LAGB are within the range of other bariatric interventions. This is even more important considering that perioperative complications in high-volume centers for LAGB placement, removal or conversion are 1-2\% [19].

These findings could lead to the consideration that LAGB could be considered as a first step for patients with high BMI; LAGB would be safer than LSG in terms of complications and conversion to RYGB [40] or MGB/OAGB [41, 42] would represent a totally reversible two-step procedure.

In summary, since LAGB is not a novel procedure, body of literature regarding this intervention is consistent, but, in our opinion, it has been misinterpreted. LAGB has been almost abandoned just because it is considered not effective or obsolete. As demonstrated in our series, LAGB is effective in long term in selected patients and more efforts should be made to find predictive selection criteria. These criteria could allow surgeons to keep the gastric banding in their armamentarium since it is safe, totally reversible and convertible to other bariatric interventions. Moreover, rate of reoperation after LAGB is in line with the percentage of reintervention after bariatric surgery; vast majority of reoperations after LAGB are removals, port-tube interventions and conversions, which are usually performed with a low rate of complications. Moreover, several factors imply in the genesis and development of obesity and that can modify the postoperative course of bariatric patients and weight loss regardless of the surgical procedure [43].
In regard of resolution of obesity related diseases, we must disclose that LAGB has never been the favourite choice for diabetic patients or subjects with metabolic syndrome in our center. Subsequently, the initial percentage of patients suffering with T2DM, HTN and dyslipidaemia was not particularly high in our cohort. Since LAGB is a purely restrictive procedure, it was not unexpected a low rate of resolution, especially in long term. Unlike other procedures [44], improvement of comorbidities after LAGB can only be related to weight loss and not to metabolic changes, indeed a $0 \%$ remission from T2DM was recorded in our study.

\section{Conclusion}

LAGB is a safe and moderately effective bariatric procedure in long term but it showed disappointing rates of removal, non-response and remission from obesity related diseases. However, LAGB could still be proposed for selected/motivated patients.

Acknowledgements Open access funding provided by Università degli Studi di Napoli Federico II within the CRUI-CARE Agreement.

Author contributions All authors performed substantial contributions to conception and design of the article and to acquisition, analysis, and interpretation of data. All authors reviewed the manuscript for important intellectual content and approved the final version for publication. All authors agree to be accountable for all aspects of the work in ensuring that questions related to the accuracy or integrity of any part of the work are appropriately investigated and resolved.

Funding We did not receive any fund or grant for this research.

\section{Compliance with ethical standards}

Conflict of interest The authors declare that they have no conflict of interest.

Ethical approval All procedures performed in this study involving human participants were in accordance with the ethical standards of the institutional and/or national research committee and with the 1964 Helsinki Declaration and its later amendments or comparable ethical standards.

Informed consent Informed consent was obtained from all the individual participants included in the study.

Open Access This article is licensed under a Creative Commons Attribution 4.0 International License, which permits use, sharing, adaptation, distribution and reproduction in any medium or format, as long as you give appropriate credit to the original author(s) and the source, provide a link to the Creative Commons licence, and indicate if changes were made. The images or other third party material in this article are included in the article's Creative Commons licence, unless indicated otherwise in a credit line to the material. If material is not included in the article's Creative Commons licence and your intended use is not permitted by statutory regulation or exceeds the permitted use, you will 
need to obtain permission directly from the copyright holder. To view a copy of this licence, visit http://creativecommons.org/licenses/by/4.0/.

\section{References}

1. Wilkinson LH, Peloso OA (1981) Gastric (reservoir) reduction for morbid obesity. Arch Surg 116:602-605

2. Kolle K, Bo O, Stadaas J (1982) Gastric banding. In: OMGI 7th Congress, Stockholm, vol 145, p 37

3. Molina M, Oria HE (1983) Gastric segmentation: a new, safe, effective, simple, readily revised and fully reversible surgical procedure for the correction of morbid obesity. In: 6th Bariatric Surgery Colloquium, Iowa City, IA, pp 2-3

4. Hallberg D, Forsell O (1985) Ballongband vid behandling av massiv överwikt. Svinsk Kirurgi 344:106-108

5. Kuzmak LI (1986) Silicone gastric banding: a simple and effective operation for morbid obesity. Contemp Surg 28:13-18

6. Vinzens F, Kilchenmann A, Zumstein V, Slawik M, Gebhart M, Peterli R (2017) Long-term outcome of laparoscopic adjustable gastric banding (LAGB): results of a Swiss single-center study of 405 patients with up to 18 years' follow-up. Surg Obes Relat Dis 13(8):1313-1319

7. Buchwald H, Oien DM (2013) Metabolic/bariatric surgery worldwide 2011. Obes Surg 23(4):427-436

8. Angrisani L, Santonicola A, Iovino P et al (2017) Erratum to: bariatric surgery and endoluminal procedures: IFSO worldwide survey 2014. Obes Surg 27(9):2290-2292

9. Angrisani L, Santonicola A, Iovino P et al (2018) IFSO worldwide survey 2016: primary, endoluminal, and revisional procedures. Obes Surg 28(12):3783-3794

10. Musella M, Milone M, Gaudioso D et al (2014) A decade of bariatric surgery. What have we learned? Outcome in 520 patients from a single institution. Int J Surg 12(Suppl 1):S183-S188. https://doi. org/10.1016/j.ijsu.2014.05.012

11. Brolin R, Kenler HA, Gorman RC et al (1989) The dilemma of outcome assessment after operations for morbid obesity. Surgery 105:337-346

12. American Diabetes Association (2010) Diagnosis and classification of diabetes mellitus. Diabetes Care 33(Suppl 1):S62-S69

13. National Institute for Health and Clinical excellence. https://www. nice.org.uk/guidance/ng136/chapter/recommendations. NICE guideline [NG136] Published date: August 2019.

14. Goldstein LB, Bushnell CD, Adams RJ et al (2011) Guidelines for the primary prevention of stroke: a guideline for healthcare professionals from the American Heart Association/American Stroke Association. Stroke 42(2):517-584

15. Beitner MM, Ren-Fielding CJ, Fielding GA (2016) Reducing complications with improving gastric band design. Surg Obes Relat Dis 12(1):150-156

16. Lyass S, Cunneen SA, Hagiike M et al (2005) Device-related reoperations after laparoscopic adjustable gastric banding. Am Surg 71(9):738-743

17. Egberts K, Brown WA, O'Brien PE (2011) Systematic review of erosion after laparoscopic adjustable gastric banding. Obes Surg 21(8):1272-1279

18. Kodner C, Hartman DR (2014) Complications of adjustable gastric banding surgery for obesity. Am Fam Physician 89(10):813-818

19. Sharples AJ, Charalampakis V, Daskalakis M, Tahrani AA, Singhal R (2017) Systematic review and meta-analysis of outcomes after revisional bariatric surgery following a failed adjustable gastric band. Obes Surg 27(10):2522-2536

20. Magouliotis DE, Tasiopoulou VS, Svokos AA, Svokos KA, Sioka E, Zacharoulis D (2017) Roux-En-Y gastric bypass versus sleeve gastrectomy as revisional procedure after adjustable gastric band: a systematic review and meta-analysis. Obes Surg 27(5):1365-1373

21. Lundberg PW, Gotsch A, Kim E, Claros L, Stotlzfus J, El Chaar M (2019) Safety of one-stage conversion surgery after failed gastric band: our experience and review of the literature. Updates Surg 71(3):445-450

22. Angrisani L, Vitiello A, Santonicola A, Hasani A, De Luca M, Iovino P (2017) Roux-en-Y gastric bypass versus sleeve gastrectomy as revisional procedures after adjustable gastric band: 5-year outcomes. Obes Surg 27(6):1430-1437

23. O'Brien PE, McPhail T, Chaston TB, Dixon JB (2006) Systematic review of medium-term weight loss after bariatric operations. Obes Surg 16(8):1032-1040

24. Naef M, MoutonWG NU et al (2010) Graft survival and complications after laparoscopic gastric banding for morbid obesity-lessons learned from a 12-year experience. Obes Surg 20(9):1206-1214

25. Mittermair RP, Obermuller S, Perathoner A et al (2009) Results and complications after Swedish adjustable gastric banding-10 years experience. Obes Surg 19(12):1636-1641

26. Froylich D, Abramovich-Segal T, Pascal G et al (2018) Long-term (over 10 years) retrospective follow-up of laparoscopic adjustable gastric banding. Obes Surg 28(4):976-980 (published correction appears in Obes Surg. 2017 Dec 1)

27. Arapis K, Tammaro P, Parenti LR et al (2017) Long-term results after laparoscopic adjustable gastric banding for morbid obesity: 18-year follow-up in a single university unit. Obes Surg 27(3):630-640

28. Shen X, Zhang X, Bi J, Yin K (2015) Long-term complications requiring reoperations after laparoscopic adjustable gastric banding: a systematic review. Surg Obes Relat Dis 11(4):956-964

29. Nguyen NT, Kim E, Vu S, Phelan M (2018) Ten-year outcomes of a prospective randomized trial of laparoscopic gastric bypass versus laparoscopic gastric banding. Ann Surg 268(1):106-113

30. Tsai C, Zehetner J, Beel J, Steffen R (2019) Long-term outcomes and frequency of reoperative bariatric surgery beyond 15 years after gastric banding: a high band failure rate with safe revisions. Surg Obes Relat Dis 15(6):900-907

31. Kowalewski PK, Olszewski R, Kwiatkowski A, Gałązka-Świderek N, Cichoń K, Paśnik K (2017) Life with a gastric band. Long-term outcomes of laparoscopic adjustable gastric banding-a retrospective study. Obes Surg 27(5):1250-1253

32. Lazzati A, De Antonio M, Paolino L et al (2017) Natural history of adjustable gastric banding: lifespan and revisional rate: a nationwide study on administrative data on 53,000 patients. Ann Surg 265(3):439-445

33. Vitiello A, Pilone V, Ferraro L, Forestieri P (2018) Is the sleeve gastrectomy always a better procedure? Five-year results from a retrospective matched case-control study. Obes Surg 28(8):2333-2338

34. Peña AS, Delko T, Couper R et al (2017) Laparoscopic adjustable gastric banding in Australian adolescents: should it be done? Obes Surg 27(7):1667-1673

35. Picot J, Jones J, Colquitt JL, Loveman E, Clegg AJ (2012) Weight loss surgery for mild to moderate obesity: a systematic review and economic evaluation. Obes Surg 22(9):1496-1506

36. Lauti M, Kularatna M, Hill AG, MacCormick AD (2016) Weight regain following sleeve gastrectomy-a systematic review. Obes Surg 26(6):1326-1334

37. Tran DD, Nwokeabia ID, Purnell S et al (2016) Revision of roux-eny gastric bypass for weight regain: a systematic review of techniques and outcomes. Obes Surg 26(7):1627-1634

38. Elder K, Wolfe B (2007) Bariatric surgery: a review of procedures and outcomes. Gastroenterology 132(6):2253-2271

39. O'Brien PE, Hindle A, Brennan L et al (2019) Long-term outcomes after bariatric surgery: a systematic review and meta-analysis of weight loss at 10 or more years for all bariatric procedures and a 
single-centre review of 20-year outcomes after adjustable gastric banding. Obes Surg 29(1):3-14

40. Zhou R, Poirier J, Torquati A, Omotosho P (2019) Short-term outcomes of conversion of failed gastric banding to laparoscopic sleeve gastrectomy or Roux-En-Y gastric bypass: a meta-analysis. Obes Surg 29(2):420-425

41. Piazza L, Di Stefano C, Ferrara F, Bellia A, Vacante M, Biondi A (2015) Revision of failed primary adjustable gastric banding to mini-gastric bypass: results in 48 consecutive patients. Updates Surg 67(4):433-437

42. Musella M, Bruni V, Greco F et al (2019) Conversion from laparoscopic adjustable gastric banding (LAGB) and laparoscopic sleeve gastrectomy (LSG) to one anastomosis gastric bypass (OAGB): preliminary data from a multicenter retrospective study. Surg Obes Relat Dis 15(8):1332-1339
43. Rossi F, Bellini G, Luongo L et al (2016) Cannabinoid receptor 2 as antiobesity target: inflammation, fat storage, and browning modulation. J Clin Endocrinol Metab 101(9):3469-3478. https:// doi.org/10.1210/jc.2015-4381

44. Del Genio G, Limongelli P, Del Genio F, Motta G, Docimo L, Testa D (2016) Sleeve gastrectomy improves obstructive sleep apnea syndrome (OSAS): 5-year longitudinal study. Surg Obes Relat Dis 12(1):70-74. https://doi.org/10.1016/j.soard.2015.02.020

Publisher's Note Springer Nature remains neutral with regard to jurisdictional claims in published maps and institutional affiliations. 